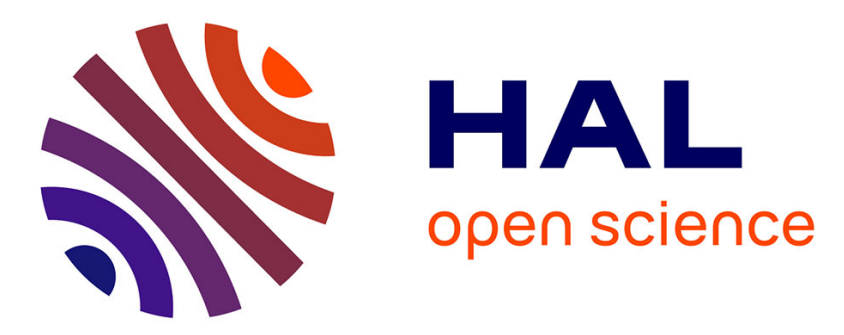

\title{
THEORETICAL MODELS AND COMPUTATION OF FAULT ENERGIES.MOMENTS DEVELOPMENTS : TRANSITION METALS AND COVALENT CRYSTALS
}

\author{
F. Ducastelle
}

\section{To cite this version:}

F. Ducastelle. THEORETICAL MODELS AND COMPUTATION OF FAULT ENERGIES.MOMENTS DEVELOPMENTS: TRANSITION METALS AND COVALENT CRYSTALS. Journal de Physique Colloques, 1974, 35 (C7), pp.C7-79-C7-90. 10.1051/jphyscol:1974707 . jpa00215863

\section{HAL Id: jpa-00215863 \\ https://hal.science/jpa-00215863}

Submitted on 1 Jan 1974

HAL is a multi-disciplinary open access archive for the deposit and dissemination of scientific research documents, whether they are published or not. The documents may come from teaching and research institutions in France or abroad, or from public or private research centers.
L'archive ouverte pluridisciplinaire HAL, est destinée au dépôt et à la diffusion de documents scientifiques de niveau recherche, publiés ou non, émanant des établissements d'enseignement et de recherche français ou étrangers, des laboratoires publics ou privés. 


\title{
THEORETICAL MODELS AND COMPUTATION OF FAULT ENERGIES.
}

\section{MOMENTS DEVELOPMENTS : TRANSITION METALS AND COVALENT CRYSTALS}

\section{F. DUCASTELLE}

Office National d'Etudes et de Recherches Aérospatiales, O. N. E. R. A., 92320 Châtıllon, France

\begin{abstract}
Résumé. - On étudie quelques propriétés reliées à la cohésion des métaux de transition et des cristaux covalents en utilisant une méthode de moments dans le cadre de l'approximation des liaisons fortes. On s'intéresse en particulier à l'énergie de cohésion et à quelques propriétés connexes : stabilité relative des structures cristallines et énergie de faute d'empilement.
\end{abstract}

\begin{abstract}
The cohesive properties of transition metals and covalent crystals are discussed in a tight-binding scheme by using the moments of the density of states. Particular attention is paid to the cohesive energy and to related properties : relative stability of crystalline structures and stacking fault energies.
\end{abstract}

1. Introduction. - In general, it is very difficult to calculate the cohesive properties of solids : cohesive energy, elastic moduli, ... The reason is that all the interactions between the charges of the system have to be taken into account. In particular the electronelectron interactions which are often treated in an approximate way are in principle very important here. Some systems are relatively simple : ionic crystals, Van der Waals solids. The simplicity in these cases lies in the fact that the total energy can be written as a sum of interatomic pair potentials. As a result, with realistic potentials the calculations are not too difficult and are in good agreement with the experimental data [1]. On the other hand, in the case of simple metals $(\mathrm{Li}, \mathrm{Na}, \ldots)$ where the electrons can be considered as free, a rather good description of the cohesive properties can be given within a pseudopotential theory [2].

When dealing now with solids where the bonds become more and more covalent, we are faced with a lot of difficulties. The wave functions of the valence electrons are very different from plane waves so that the pseudopotential theory is inapplicable, at least in its simplest form. Another well-known approach is then to write down the wave function as a linear combination of atomic orbitals. This is the LCAO or tight-binding method. It is at once apparent that the electronic energy calculated in this scheme depends on the crystalline structure. The situation is to be compared with the case of simple metals for which, in a first approximation, the electronic energy reduces to the energy of the free electron gas and is therefore independent of the structure for a given density. The main advantage of the tight-binding approximation is that it enables us to compare directly the total energy of the solid with the free atom energy, so that it is a good approximation to neglect all contributions except those coming from the variation of the one-electron energies. The problem is therefore to calculate the electronic spectrum. Large progresses have been made in the field of band structure computations during these last ten years, and there is no basic difficulty in using these calculations to obtain the electronic band energy $E_{\mathrm{b}}$. However it is clear that in this procedure we start from an information which is too rich. All the fine details of the electronic structure are generally irrelevant when calculating the energy $E_{\mathrm{b}}$ :

$$
E_{\mathrm{b}}=\int^{E_{\mathrm{F}}} E n(E) \mathrm{d} E
$$

where $n(E)$ is the density of states and $E_{\mathrm{F}}$ the Fermi energy..In short one can say that we are only interested in the integral properties of the density of states. Now we can get some information on $n(E)$ from its moments $\mu_{n}$ :

$$
\mu_{n}=\int E^{n} n(E) \mathrm{d} E .
$$

The first moments are sufficient to characterize in a rough way a positive function like $n(E)$. We can therefore simulate the behaviour of $n(E)$ by using a function which has the same first moments, and from the definition of the moments it is clear that this simulation will be mainly effective when calculating integrals over the density of states. What makes this program attractive in our case is that the first moments can easily be calculated in the tight-binding approximation. In this way we shall avoid the intermediate 
trip among band structure calculations. The possible accuracy loss will be more than compensated by the simplicity of the method and by other advantages. The first one is that the moments are obtained as explicit expressions in terms of the basic parameters which enter the theory. The discussion of the dependance of the cohesive properties on these parameters will therefore be easy. The second advantage is that in some cases we shall be able to calculate directly variations of cohesive energies instead of comparing two large quantities. Finally this method does not rely on the assumption of lattice periodicity so that it can be applied to defects and more generally to disordered structures as well.

Let us now give an outline of this article. In section 2 we recall the main features of the tight-binding approximation. We give the general principles for the calculation of moments and we describe some properties of these moments. In section 3 we apply the moments technique to transition metals. We calculate the cohesive energy and compare the different crystalline structures. Particular attention is paid to the HCP-FCC comparison, which leads to a discussion of the stacking fault energies in these structures. Finally the elastic properties of transition metals are briefly mentioned. In section 4 a similar discussion is given concerning covalent crystals such as diamond, silicon and germanium.

\section{Moments of the density of states in the tight-} binding approximation. -2.1 THE TIGHT-BINDING APPROXIMATION. - We shall describe here the simplest form of the tight-binding approximation by assuming that there is a single s atomic state for each free atom. The extension to actual situations is easy and will be given in sections 3 and 4 . The hamiltonian of the crystal is :

$$
H=T+\sum_{i} V_{i}
$$

where $T$ is the kinetic energy and $V_{i}$ the atomic potential centred on site $i$. The atomic orbitals

$\langle\mathbf{r} \mid i\rangle=\varphi\left(\mathbf{r}-\mathbf{R}_{i}\right)$ are assumed to be sufficiently concentrated around the nuclei $i$ for the overlap $\langle i \mid j\rangle$ to be negligible :

$$
\langle i \mid j\rangle=\int \mathrm{d} \mathbf{r} \varphi\left(\mathbf{r}-\mathbf{R}_{i}\right) \varphi\left(\mathbf{r}-\mathbf{R}_{j}\right)=\delta_{i j} .
$$

In this way the states $\mid i>$ form a complete orthonormal basis for the electronic states, and an eigenstate $|\psi\rangle$ of $H$ can be written as :

$$
|\psi\rangle=\sum_{i} a_{i}|i\rangle
$$

We now use the fact that the states $|i\rangle$ are the eigenstates of the atomic hamiltonians $H_{i}=T+V_{i}$ :

$$
\left.\left(T+V_{i}\right)\left|i>=E_{0}\right| i\right\rangle
$$

where $E_{0}$ is the atomic d level. From (2.1) and (2.4) we obtain :

$$
\begin{aligned}
\langle i|H| i\rangle & =E_{0}+\alpha \\
\alpha & =\sum_{k \neq i}\left\langle i\left|V_{k}\right| i\right\rangle .
\end{aligned}
$$

$\alpha$ gives a measure of the crystalline field at site $i$ and produces a shift of the energies. The off-diagonal terms are given by :

$$
\begin{aligned}
\beta_{i j}=\langle i|H| j\rangle & =\left\langle i\left|\sum_{k \neq j} V_{k}\right| j\right\rangle \\
& \simeq\left\langle i\left|V_{i}\right| j\right\rangle
\end{aligned}
$$

where we have neglected three-centre integrals. Because of the rapid decrease of $\beta_{i j}$ when the distance $\left|\mathbf{R}_{j}-\mathbf{R}_{i}\right|$ increases, we usually only take into account these transfer integrals between first neighbours. We therefore have a single negative integral $\beta$. In the case of a periodic lattice with one atom per cell, the eigenstates of $H$ are given by the following Bloch functions :

$$
\left|\psi_{k}>=\frac{1}{\sqrt{N}} \sum_{i} \mathrm{e}^{i \mathbf{k} \cdot \mathbf{R}_{i}}\right| i>
$$

where $N$ is the number of atoms. The eigenvalues $\varepsilon_{k}$ of He are then given by :

$$
\varepsilon_{k}=\beta \sum_{\mathbf{R}}^{\prime} \mathrm{e}^{i \mathbf{k} \cdot \mathbf{R}}+\alpha+E_{0}
$$

where the prime means that the summation is restricted to the first neighbours.

2.2 MOMENTS OF THE DENSity of STATES [3]. - The moment of order $n$ of the density of states, $\mu_{n}$, is defined by :

$$
\mu_{n}=\int E^{n} n(E) \mathrm{d} E .
$$

The density of states $n(E)$ is given by :

$$
n(E)=\frac{1}{N} \sum_{k} \delta\left(E-\varepsilon_{k}\right)=\frac{1}{N} \operatorname{Tr} \delta(E-H)
$$

and finally

$$
\begin{aligned}
\mu_{n} & =\frac{1}{N} \int E^{n} \operatorname{Tr} \delta(E-H) \mathrm{d} E \\
& =\frac{1}{N} \operatorname{Tr} H^{n} .
\end{aligned}
$$

Being expressed as a trace the moments $\mu_{n}$ can be calculated in any orthonormal basis. In particular we may choose the atomic basis :

$$
\mu_{n}=\frac{1}{N} \sum_{i}\left\langle i\left|H^{n}\right| i\right\rangle
$$


Inserting now the identity operator $I=\sum_{j}|j\rangle\langle j|$ in eq. (2.12) we get :

$$
\begin{aligned}
\mu_{n} & =\frac{1}{N} \sum_{i, j}\langle i|H| j\rangle\left\langle j\left|H^{n-1}\right| i\right\rangle \\
& =\frac{1}{N} \sum_{i_{1}, \ldots, i_{n}}\left\langle i_{1}|H| i_{2}\right\rangle \cdots\left\langle i_{n}|H| i_{1}\right\rangle .
\end{aligned}
$$

For a periodic lattice with one atom per cell, we obtain at once the first moments :

$$
\begin{aligned}
& \mu_{1}=\alpha+E_{0} \\
& \mu_{2}=z \beta^{2}
\end{aligned}
$$

where $z$ is the coordination number of the lattice.

We check that $\mu_{1}=\alpha+E_{0}$ gives the shift of the band. On the other hand $\sqrt{\mu_{2}}$ gives a measure of the width of the band, proportional to $|\beta|$ as expected. Let us use now centred moments (i. e. the zero of energy is such that $E_{0}+\alpha=0$ ). Each factor in the right hand side of (2.13) corresponds to a step from site $i_{p}$ to the neighbouring site $i_{p+1}$. In the particular model considered here a factor $\beta$ is associated to each step, so that :

$$
\mu_{n}=\frac{\beta^{n}}{N} \sum_{i} P_{i}(n)
$$

where $P_{i}(n)$ is the number of closed paths of $n$ steps starting from site $i$. If all sites are equivalent (2.15) reduces to :

$$
\mu_{n}=\beta^{n} P(n) .
$$

As an example, let us consider a linear chain. Eq. (2.16) leads to :

$$
\mu_{2 n}=\frac{2 n !}{n !^{2}} \beta^{2 n} \quad \mu_{2 n+1}=0 .
$$

It is easily checked that the same result would have been obtained by using the well known density of states of the linear chain :

$$
\mu_{n}=\int E^{n} \frac{1}{\pi} \frac{1}{\sqrt{E^{2}-4 \beta^{2}}} \mathrm{~d} E .
$$

2.3 Properties OF THE MOMENTS. - When the moments are known, the density of states can in principle be obtained from its characteristic function $f(x)$ :

$$
f(x)=\sum_{n} \frac{(-i x)^{n}}{n !} \mu_{n} .
$$

Actually $f(x)$ is the Fourier transform of $n(E)$; hence :

$$
n(E)=\frac{1}{2 \pi} \int \mathrm{e}^{i x E} f(x) \mathrm{d} x .
$$

It must be emphasized that the series (2.19) is not a perturbation series: the moments are never negli- gible ; as a matter of fact the integration of a particular term of $f(x)$ gives a derivative of the Dirac $\delta$-function. In the case of a linear chain, we insert the moments given by (2.17) in eq. (2.19) ; the characteristic function is the Bessel function of zero order which is indeed the Fourier transform of the density of states.

In periodic structures the density of states exhibit some singularities called Van Hove singularities, which are connected to the asymptotic behaviour of the characteristic function. Band edges are particular singularities and they can be obtained from the asymptotic form of the moments. Let $M$ be the largest eigenvalue in absolute value, then :

$$
M=\lim _{n \rightarrow \infty} \mu_{n}^{1 / n} .
$$

A more detailed discussion of the properties of moments is given for example in references [3] and [4].

2.4 RECONSTRUCTION OF THE DENSITY OF STATES [4], [5]. -- When using a moments technique, the main problem is to be able to build up a reasonable density of states from the knowledge of a finite number of moments. Since this problem is undeterminate a lot of methods are available, and the best choice among them will depend on the problem at hand. If we have only a small number of moments we shall use simple trial functions. For example with only $\mu_{1}$ and $\mu_{2}$ the choice of a gaussian is quite natural. With some moments more, we may try to correct the gaussian, and this can indeed be done through a so-called Edgeworth expansion. These functions of course cannot simulate in a realistic way the density of states itself, but as said before, they are probably convenient when calculating integrals over the density of states. A drawback of this method is that it leads to infinite tails in the density of states so that corrections due to high order moments may only affect unphysical regions, in which case other methods can be used. For example if we have a guess about the position of band edges, we can try a family of orthonormal polynomials such as Legendre polynomials. Now the difficulty is that we often get spurious oscillations and regions of negative densities of states. In fact when dealing with bounded spectra, it seems that the best method is to use continuous fraction expansions [4], [5], [6].

There is still an important question : what is the error made by using for the density of states an approximate curve fitted to a finite number of moments. Actually there is no answer to this question. Bounds for the error can only be obtained for the integrated density of states $N(E)$ :

$$
N(E)=\int^{E} n(x) \mathrm{d} x .
$$

As for the density of states itself, we shall just mention here a theorem which will be used in the following [7]. Let $f(E)$ and $g(E)$ be two functions vanishing outside a given interval, and which have their $n$ first moments 
equal, then the function $h=f-g$ has at least $n+1$ zeros within the interval and the function $h_{p}$ defined by :

$$
h_{p}(t)=\int^{t} h_{p-1}(x) \mathrm{d} x \quad h_{0}=h
$$

has at least $n+1-p$ zeros. In the case where $g$ is an approximate curve for $f$, we see that $g$ must oscillate around $f$, but it is not possible to give a priori a bound for the amplitude of the oscillations.

3. Transition metals. - 3.1 InTRODUCTION. Transition metals are characterized by the progressive filling along a series of the inner $d$ states. In the solid these electrons are still well concentrated around the nuclei and can then be described from a basis of atomic $\mathrm{d}$ orbitals. On the other hand the $\mathrm{s}$ wave functions are much more spread out and give rise in the solid to a broad band of free-like electrons. The $\mathrm{d}$ and $\mathrm{s}$ bands overlap, and actually we can no more speak of pure d or $\mathrm{s}$ states; s-d mixing effects are indeed most important when studying transport properties for example.

As far as the cohesive properties are concerned, it is nevertheless clear that $d$ electrons play the most important part. For example one can observe that there is almost one order of magnitude of difference between the cohesive energies of transition metals and those of simple metals. In the following we shall therefore neglect everywhere s electrons and s-d mixing effects, and we shall treat $d$ electrons within the tightbinding approximation.

3.2 THE TIGHT-BINDING APPROXIMATION FOR TRANSITION METALS. - 3.2.1 Description of the model. When going from our oversimplified model of $\S 2.1$ to the actual case of transition metals, the main complication which arises is due to the degeneracy of $d$ states : we have now five orbitals per site. We choose the usual real basis :

$$
\begin{aligned}
& \varphi_{1}(r)=\frac{x y}{r^{2}} f(r) ; \quad \varphi_{2}(r)=\frac{y z}{r^{2}} f(r) ; \quad \varphi_{3}(r)=\frac{z x}{r^{2}} f(r) \\
& \varphi_{4}(r)=\frac{x^{2}-y^{2}}{2 r^{2}} f(r) ; \varphi_{5}(r)=\frac{3 z^{2}-r^{2}}{2 \sqrt{3} r^{2}} f(r) .
\end{aligned}
$$

The crystalline field integrals $\alpha_{i}^{\lambda}$ and the transfer integrals $\beta_{i j}^{\lambda \mu}$ are now defined by :

$$
\begin{aligned}
& \alpha_{i}^{\lambda}=\left\langle i, \lambda\left|\sum_{j \neq i} V_{j}\right| i, \lambda\right\rangle \\
& \beta_{i j}^{\lambda \mu}=\left\langle i, \lambda\left|V_{i}\right| j, \mu\right\rangle \quad \lambda, \mu=1, \ldots, 5 .
\end{aligned}
$$

When dealing with periodic structures the solution of the Schrödinger equation is given from the diagonalization of a $5 \times 5$ matrix (if there is one atom per cell) which yields five bands $\varepsilon_{k, \alpha}, \alpha=1, \ldots, 5$. Then the first thing to do is to reduce the number of independent parameters. The crystalline field integrals are usually assumed to be very small as compared to the transfer ones, the reason being that they involve the asymptotic part of the potential of neighbouring atoms [8]. Moreover they are probably cancelled to a large extent by contributions of opposite sign [9]. In a first approximation we shall therefore neglect these integrals. Let us now consider the transfer integrals $\beta_{i j}^{\lambda \mu}$. We fix the $z$ axis along $\mathbf{R}_{j}-\mathbf{R}_{i}$. It is then easily realized that we only obtain three different integrals :

$$
\begin{aligned}
& \beta^{55}=\mathrm{dd} \sigma ; \quad \beta^{44}=\beta^{11}=\mathrm{dd} \pi \\
& \beta^{22}=\beta^{33}=\mathrm{dd} \delta .
\end{aligned}
$$

Due to the form of the $d$ orbitals (for example $\varphi_{5}$ points along $O z$ ) we see also at once that :

$$
\begin{gathered}
\mathrm{dd} \sigma<0 ; \quad \mathrm{dd} \pi>0 ; \quad \mathrm{dd} \delta<0 \\
|\mathrm{dd} \delta| \ll \mathrm{dd} \pi, \quad|\mathrm{dd} \sigma| .
\end{gathered}
$$

The general expression of $\beta_{i j}^{\lambda \mu}$ for an arbitrary choice of axis is then obtained by performing a rotation in the space of $d$ orbitals. It depends linearly on the three integrals $\mathrm{dd} \sigma, \mathrm{dd} \pi$ and $\mathrm{dd} \delta$, and on the director cosines of $\mathbf{R}_{j}-\mathbf{R}_{i}$. This expression is given in reference [10].

3.2.2 Moments. - The extension of eq. (2.13) is straightforward :

$$
\begin{array}{r}
\mu_{n}=\frac{1}{5 N} \sum_{\substack{i_{1}, \ldots, i_{n} \\
\lambda_{1}, \ldots, \lambda_{n}}}<i_{1}, \lambda_{1}|H| i_{2}, \lambda_{2}>\cdots \\
\quad \cdots<i_{n}, \lambda_{n}|H| i_{1}, \lambda_{1}>
\end{array}
$$

where $N$ is now the number of sites and $5 N$ the total number of states. In this way $n(E)$ is normalized to unity : $\mu_{0}=1$.

Defining then the $5 \times 5$ matrices $H_{i j}$ by :

$$
H_{i j}=\sum_{\lambda, \mu} \mid i, \lambda>\langle i, \lambda|H| j, \mu><j, \mu|
$$

we get :

$$
\mu_{n}=\frac{1}{5 N} \sum_{i_{1}, i_{2}, \ldots, i_{n}} \operatorname{Tr} H_{i_{1} i_{2}} \ldots H_{i_{n} i_{1}}
$$

where now the trace is only taken over the degeneracy indices $\lambda$. As in $\S 2.2$ the sum over site indices is equivalent to a sum over closed paths, but now the contribution of a particular path depends on its geometry. This is to be related to the anisotropy of $d$ states : the mathematical counterpart being that we deal with products of non commuting matrices.

Let us apply (3.7) to the case of a lattice with one atom per cell. Since the shift of the band has been neglected $\mu_{1}=0$, and $\mu_{2}$ is given by:

$$
\mu_{2}=\frac{1}{5} \sum_{j} \operatorname{Tr} H_{i j}^{2}
$$

where the sum is performed over the first neighbours of site $i$. Let us calculate the contribution of a particular term in the right hand side of (3.8). We can choose the 
axes at will; we take therefore $\mathrm{Oz}$ along $\mathbf{R}_{J}-\mathbf{R}_{i}$, and from (3.2) we obtain :

$$
\operatorname{Tr} H_{i j}^{2}=\mathrm{dd} \sigma^{2}+2 \mathrm{dd} \pi^{2}+2 \mathrm{dd} \delta^{2}
$$

and finally:

$$
\mu_{2}=\frac{z}{5}\left(\mathrm{dd} \sigma^{2}+2 \mathrm{dd} \pi^{2}+2 \mathrm{dd} \delta^{2}\right)
$$

Considering higher moments requires the knowledge of the actual crystalline structure. $\mu_{3}$ is related to the three-steps paths; therefore $\mu_{3}=0$ in a BCC lattice if we only take into account the first neighbours (actually $\mu_{2 n+1}=0$ for all $n$ in this case). But of course $\mu_{3} \neq 0$ in the FCC structure [7].

3.2.3 Numerical values of the parameters. - First principles calculations of the integrals $\mathrm{dd} \sigma, \mathrm{dd} \pi$ and $\mathrm{dd} \delta$ are rather difficult. These integrals involve indeed the long range part of the atomic wave functions and of the potential, and it is obvious that it is precisely in these regions that the use of pure atomic functions is open to criticism. Nevertheless such a calculation can be tried by using for example the simple Slater's rules to write down the d wave function and the atomic potential [7]. The results for $\mathrm{dd} \sigma$ and $\mathrm{dd} \pi$ are given in figure $1, \operatorname{dd} \delta$ being always negligible. (In this calculation the interatomic distance corresponds to a $\mathrm{BCC}$ structure.)

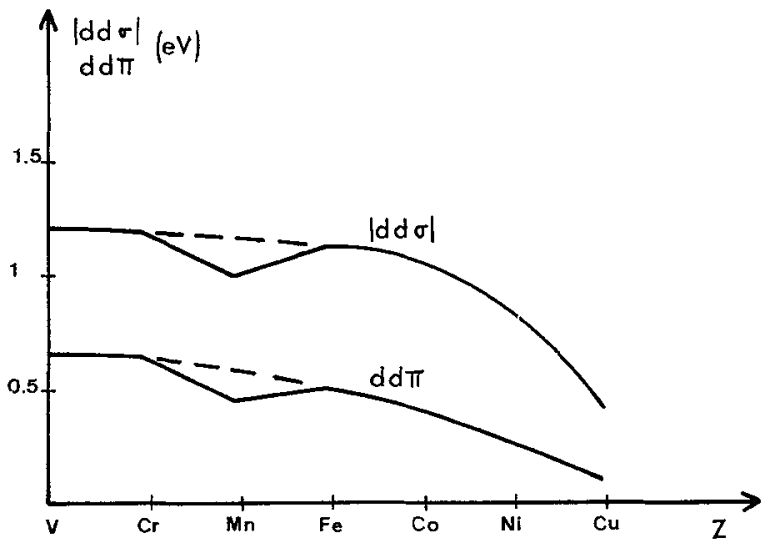

FIG. 1. - Transfer integral for the $3 \mathrm{~d}$ series (BCC structure ; first neighbours).

Another approach for estimating these integrals is to use the so-called interpolation schemes. The band structure calculations for transition metals are not usually done in the tight-binding approximation, but from more sophisticated methods which are in principle more reliable as far as the numerical results are concerned. Generally it is found that these calculations can be more or less reproduced by using the tightbinding expressions for $\varepsilon(\mathbf{k})$ where the transfer integrals are fitted in the best possible way. This provides us with other values for $\mathrm{dd} \sigma$ and $\mathrm{dd} \pi$. As a whole they agree with our values provided we apply to the latter a coefficient around 0.7 .

The general trend of the transfer integrals as pictured in figure 1 can in fact be explained from simple physical arguments. When going along a series, the ionic potential seen by a d electron is more and more important due to the weak screening provided by the other $d$ electrons. As a consequence, the atomic d orbitals are more and more concentrated and their overlap decreases. When going now from the $3 \mathrm{~d}$ to the $4 \mathrm{~d}$ and $5 \mathrm{~d}$ series, the atomic orbitals are more and more spread out and the overlap increases. This explains the behaviour of the band width shown in figure 2 . The

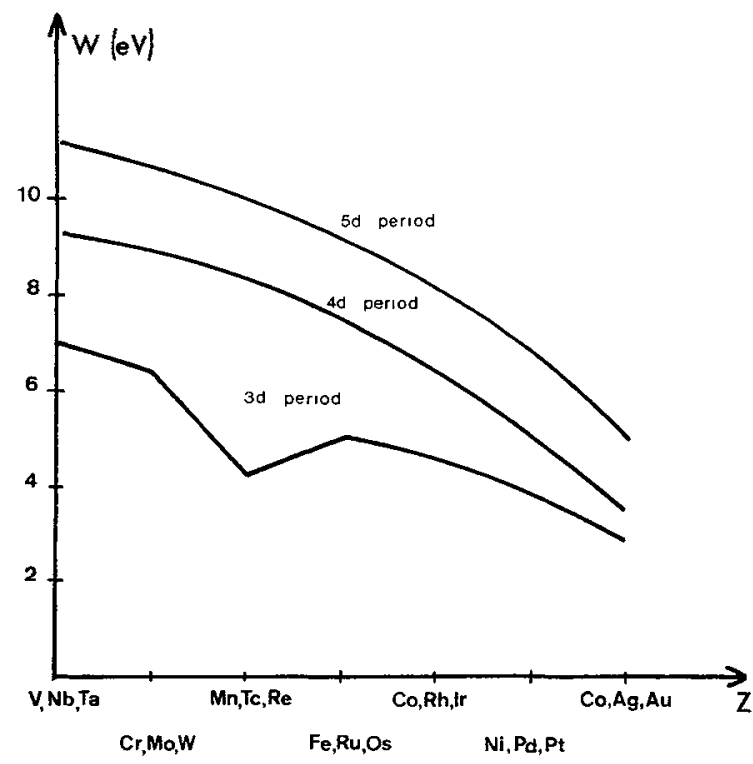

Fig. 2. - Width of the d band of transition metals. (From various calculations; see the review by Dimmock [11].)

actual bandwidth depends on $\mathrm{dd} \sigma, \mathrm{dd} \pi$ and on the crystalline structure. An empirical rule is that the bandwidth $W$ is given by [9] :

$$
W \simeq 4 \sqrt{\mu_{2}} .
$$

When studying FCC or HCP structures first principles calculations and interpolation schemes agree to conclude that the transfer integrals between second neighbours are negligible. This is no more true for BCC structures, in which case transfer integrals for second neighbours are about one half of those for first neighbours. When this is taken into account one finds that the bandwidth as given by $(3.11)$ is very similar to the FCC one.

\subsection{COHESIVE ENERGY AND RELATIVE STABILITY} OF CRYSTALLINE STRUCTURES. - 3.3.1 Cohesive energy [8], [7]. - The variation of the cohesive energy of transition metals with the filling of the $\mathrm{d}$ band is very regular, at least for the $4 \mathrm{~d}$ and $5 \mathrm{~d}$ series (Fig. 3). The variation is roughly parabolic with a maximum 


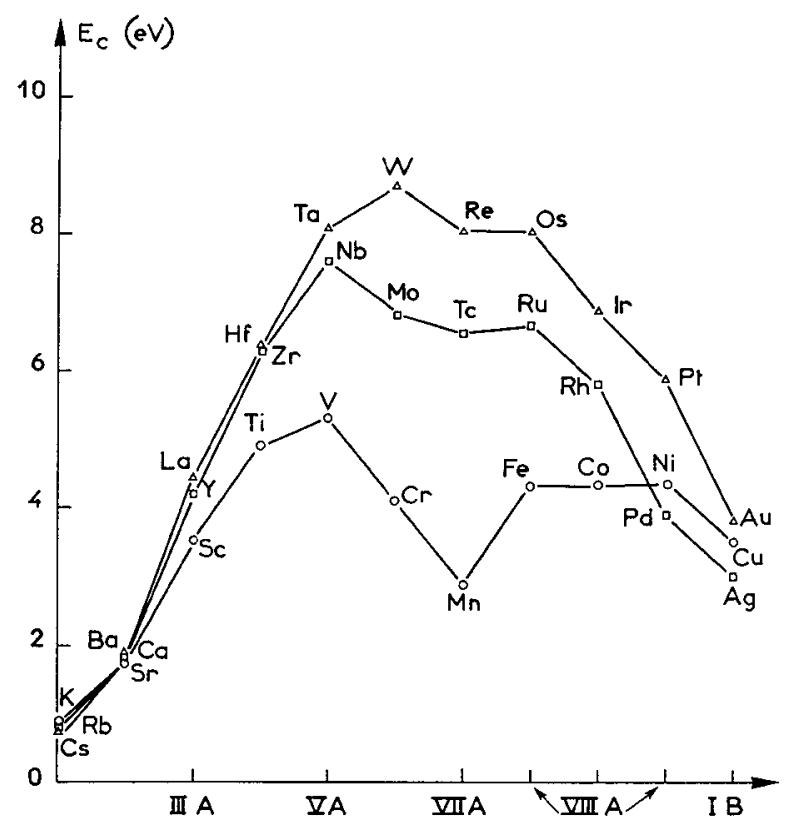

FIG. 3. - Experimental values of the cohesive energy $E_{\mathrm{c}}$.

value of the order of $8 \mathrm{eV} / \mathrm{atom}$. This can be explained qualitatively in a very simple way [8]; due to the broadening of the electronic spectrum when going from the free atoms to the crystal, bonding states can be filled until the middle of the series is reached; then antibonding states are filled and the cohesive energy decreases. An implicit assumption in the argument is that the cohesive energy can be calculated from the sum of the one-electron energies :

$$
-E_{\mathrm{c}}=10 \int^{E_{\mathrm{F}}}\left(E-E_{0}\right) n(E) \mathrm{d} E
$$

where the factor 10 accounts for the total degeneracy (spin included). It can be objected that the true electronic contribution to $E_{\mathrm{c}}$ must be corrected by the electron-electron interactions which are counted twice in a pure Hartree scheme. Actually eq. (3.12) can be justified in the context of our tight-binding approximation [8], [12].

Now we can estimate $E_{\mathrm{c}}$. A first simple approximation will be to simulate $n(E)$ by a gaussian fitted to $\mu_{2}$ :

$$
n(E)=\frac{1}{\sqrt{2 \pi \mu_{2}}} \mathrm{e}^{-E^{2} / 2 \mu_{2}}
$$

which leads to :

$$
E_{\mathrm{c}}=10 \sqrt{\frac{\mu_{2}}{2 \pi}} \mathrm{e}^{-E_{\mathrm{F}}^{2} / 2 \mu_{2}} .
$$

The variation of $E_{\mathrm{c}}$ along the transition series is given in figure 4. (Notice that both $\mu_{2}$ and $E_{\mathrm{F}}$ are varying with the number of $d$ electrons.) Let us give some comments on these results :

- First it is clear that the calculated $E_{\mathrm{c}}$ is too weak at the top of the $d$ band. Despite its filled $d$ band,

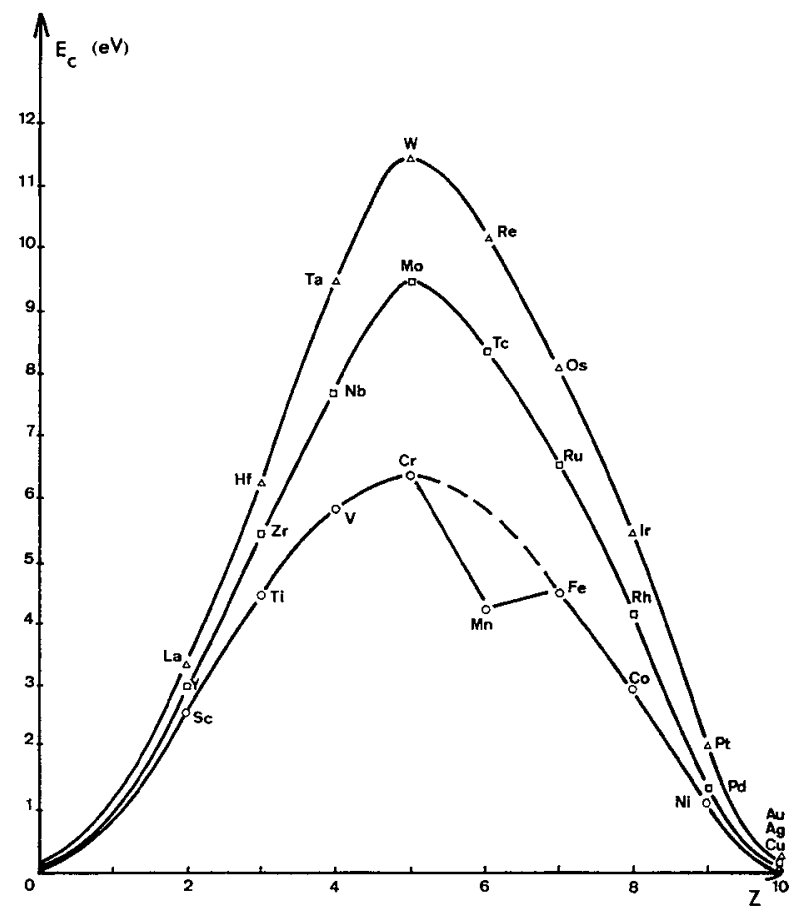

FIG. 4. - Theoretical results for the cohesive energy $E_{\mathrm{c}}$.

copper has a rather large cohesive energy. A simple perturbation calculation shows that the s-d mixing can provide the missing terms [8].

- Another point is that the results are not very good for the $3 \mathrm{~d}$ series which is unfortunately the more interesting from a metallurgical point of view. The simultaneous appearance of magnetism for these metals suggest that correlation effects have not been properly taken into account. Up to now there is no simple method to correct these results. Among these metals, manganese is more particular still in all its cohesive properties. Nevertheless its low $E_{\mathrm{c}}$ is consistent with its anomalous large parameter and therefore with its weak transfer integrals (see Fig. 1-4).

- Finally spin-orbit interactions which are expected to be rather important for the $5 \mathrm{~d}$ series have been completely neglected.

As a whole and due to the rough approximations made throughout the calculations, the results are rather good and give a strong support to the tightbinding description of transition metals.

3.3.2 Comparison of different crystalline structures [7]. - 3.3.2.1 Generalities. - As shown in table I, transition metals crystallize in the simple structures BCC, FCC or HCP (an exception is manganese which exhibits several complicated structures), and the succession of these structures is quite regular, suggesting that here again the important parameter is the filling of the $d$ band, $Z$. Because allotropic transformations exist for some metals below the melting point, variations $\delta E_{\mathrm{c}}$ of cohesive energies from 
TABLE I

Crystalline structures of transition metals. $\mathrm{Mn}$ and $\mathrm{Fe}$ are apart; they exhibit several cubic structures

\begin{tabular}{|c|c|c|c|c|c|c|c|c|}
\hline CFC & $\mathrm{HCP}$ & \multicolumn{3}{|c|}{$\mathrm{BCC}$} & \multicolumn{2}{|c|}{$\mathrm{HCP}$} & \multicolumn{2}{|c|}{ CFC } \\
\hline & & $\mathrm{T}_{1}$ & V & $\mathrm{Cr}$ & Mn & tee & Co & $\mathrm{Ni}$ \\
\hline & & $\mathrm{Zr}$ & $\mathrm{Nb}$ & Mo & Tc & Ru & Rh & $\mathrm{Pd}$ \\
\hline & & $\mathrm{Hf}$ & $\mathrm{Ta}$ & W & $\operatorname{Re}$ & Os & Ir & $\mathrm{Pt}$ \\
\hline
\end{tabular}

one structure to another are expected to be rather weak. By using thermodynamic data, Kaufman found indeed that these variations are about $10^{-2}$ to $10^{-1} \mathrm{eV}$, which yields relative values about $10^{-3}, 10^{-2}$ [13]. The situation seems therefore hopeless; all the approximations made in calculating $E_{\mathrm{c}}$ imply uncertainties around $10^{-1} \mathrm{eV}$. This explains why calculations based on the comparison of two large values of $E_{\mathrm{c}}$ obtained from band structure computations are either doubtful or difficult [14]. Let us consider for example the FCCBCC comparison; both lattices are rather different: for a given atomic volume the first neighbours are not at the same distance; the number of first neighbours is different; finally second neighbours are important in the BCC lattice. Even at the stage of the second moments $\mu_{2}$ the comparison is therefore difficult. The only thing to do is to check that their values are actually close together, which is indeed the case when using the numerical values given in $\S 3.2 .4$. This is important since it was said in $\S 1$ that the densities of states of transition metals are strongly dependent of the crystalline structure. The point is that the gross features of $n(E)$ are not very different from one structure to another so that the integral properties of $n(E)$ ( $\mu_{2}$ values for example) are not very sensitive to the atomic arrangement when the volume is held fixed.

The conclusion of this section is that no convincing argument can be put forward to explain the particular stability of the BCC structure in the middle of the transition series.

\subsubsection{Comparison of the FCC and HCP struc-} tures. - In spite of the smaller relative variation in cohesive energy $\delta E_{\mathrm{c}}$, the situation is better when comparing FCC and HCP structures. In both structures indeed the local environment of the atoms is very similar; first and second neighbours are at the same distance implying that we have not to bother about variations of the transfer integrals with the distance. Thus moments can be directly compared. In the following we extend the discussion to the case of any compact structure obtained by stacking close packed planes. An important topological property of these structures is that the numbers of closed paths of $n$ steps are the same [15]. As a consequence the moments and the densities of states are equal for all these structures. When introducing the degeneracy of $d$ states this result is no more true; as explained before the geometry of the paths will be involved. To distinguish among different structures, a path must visit at least three neighbouring close packed planes. This implies at least four steps. Thus the moments are still equal up to $\mu_{3}$ and we can assume that the values of $\mu_{4}$ will characterize the structures. An important parameter is therefore $\delta \mu_{4}$ :

$$
\delta \mu_{4}=\mu_{4}(\mathrm{FCC})-\mu_{4}(\mathrm{HCP}) .
$$

$A$ priori $\delta \mu_{4}$ depends on dd $\sigma$ and dd $\pi$. Yet it can be shown that the sign is fixed [16] :

$$
\delta \mu_{4} \geqslant 0 \text {. }
$$

We have now to calculate cohesive energies by fitting curves to their four first moments. This gives us $\delta n(E)$, and the variation $\delta E_{\mathrm{c}}$ :

$$
\delta E_{\mathrm{c}}=-\delta \int^{E_{\mathrm{F}}} E n(E) \mathrm{d} E=\int^{E_{\mathrm{F}}} \mathrm{d} E \int^{E} \mathrm{~d} x \delta n(x) .
$$

(To obtain (3.18) one must take into account the variation of $E_{\mathrm{F}}$.) From the theorem mentioned in $\S 2.4$, we can now assert that $\delta E_{\mathrm{c}}$ must have at least two zeros when filling the $d$ band. If it has actually only two zeros, the positive sign of $\delta \mu_{4}$ implies that

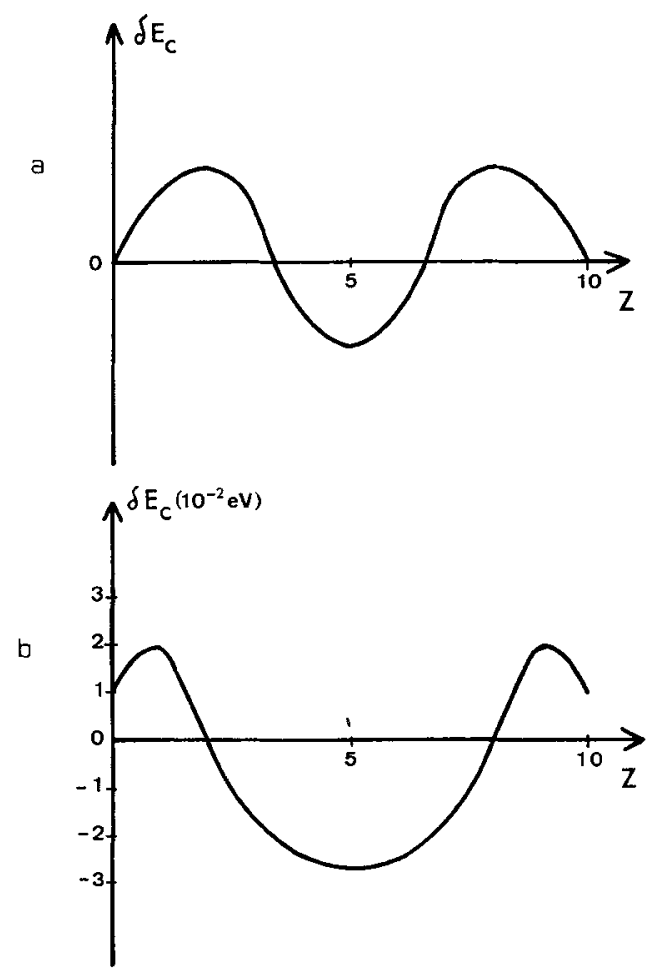

FIG. $5 a .-$ Qualitative variation of $\delta E_{\mathrm{e}}=E_{\S}(\mathrm{FCC})-E_{\mathrm{c}}(\mathrm{HCP})$ as a function of the filling of the $d$ band.

FIG. 5b. - Quantitative results obtained from an Edgeworth expansion. 
$\delta E_{\mathrm{c}}$ is positive at the bottom and the top of the band (Fig. 5a). Numerical results have been obtained by using an Edgeworth expansion for $n(E)$, and are reproduced in figure $5 b$. The maximum value of $\delta E_{\mathrm{c}}$, $\left|\delta E_{\mathrm{c}}\right|_{\max } \simeq 5 \times 10^{-2} \mathrm{eV}$ is in good agreement with Kaufman's estimates. We also find that FCC is stabilized at the edges of the band, which is indeed the case (see table I).

\subsubsection{Stacking fault energies in compact structures.} - The previous calculations can obviously be extended to the case of stacking faults. Let us consider a particular compact structure and define $\Delta \mu_{4}$ by :

$$
\Delta \mu_{4}=\mu_{4}-\mu_{4}(\mathrm{FCC}) \text {. }
$$

By looking at the paths in this structure, it is clear that $\Delta \mu_{4}$ is proportional to $\delta \mu_{4}$, the coefficient depending on the nature and on the number of faults with respect to the FCC lattice. Stacking faults in the HCP structure are studied in a similar way. The same coefficient is found for intrinsic and extrinsic faults. Finally, if we assume that the variations in cohesive energies are proportional to $\Delta \mu_{4}$, we obtain for the stacking fault energy $\gamma$ :

$$
\gamma=p / D \delta E_{\mathrm{c}}
$$

where $D$ is the number of close packed planes and $p$ a coefficient whose value is 2 for intrinsic and extrinsic faults [7]. Using the maximum value of $\left|\delta E_{\mathrm{c}}\right|$, we find :

$$
\gamma_{\max } \simeq 300 \mathrm{erg} / \mathrm{cm}^{2} \text {. }
$$

The variation of $\gamma$ with the filling of the $d$ band is sketched in figure 6 whereas experimental values for $\mathrm{NiCo}$ and $\mathrm{NiCu}$ alloys are shown in figure 7 [17].

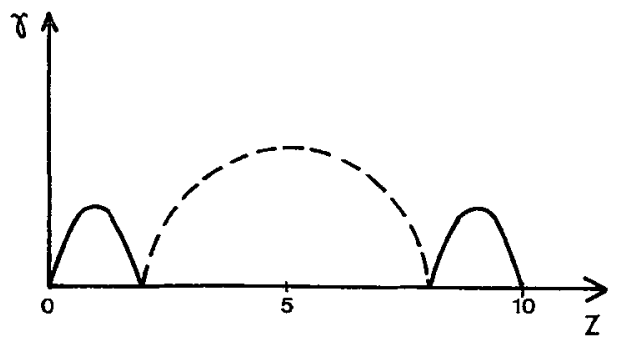

FIG. 6. - Qualitative variation of the stacking fault energy fault in a FCC lattice; ..... : fault in a HCP structure.

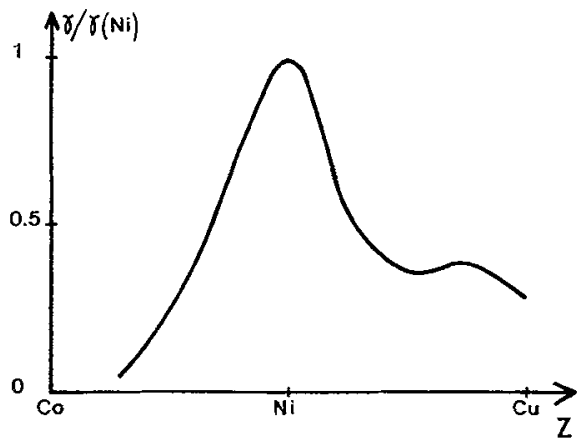

Fig. 7. - Experimental values of $\gamma$ for $\mathrm{NiCo}$ and $\mathrm{NiCu}$ alloys [17]
Nickel is a strong ferromagnet, $i$. e. the subband corresponding to one spin direction is full. As a consequence $\delta E_{\mathrm{c}}$ and $\gamma$ must be divided by two, giving a value for $\gamma$ around $150 \mathrm{erg} / \mathrm{cm}^{2}$, which is not unreasonable.

Variations $\Delta \mu_{4}$ were found to be the same for intrinsic and extrinsic faults. Of course this property is no more true for higher moments, and there is no reason why the corresponding stacking fault energies should be the same. The difference must oscillate around zero when filling the $\mathrm{d}$ band, but the amplitude of the oscillations may be important. Actually some evidences of measurable differences have been reported recently [18].

3.4 ELASTIC PROPERTIES. - The model described in previous sections cannot be used directly for calculating the elastic properties of transition metals. To see that let us introduce an uniform compression of the solid. The transfer integral increase; and so does the cohesive energy. Thus there is no repulsive terms in our model. These terms would appear through a more detailed treatment of the contributions neglected in eq. (3.12). Up to now no attempt has been made to calculate directly this term; this is very difficult indeed and a first convenient approximation is to use an empirical Born-Mayer repulsive interatomic potential whose parameters are fitted in order to reproduce the true atomic volume and the compressibility. For a complete discussion we refer to [19] and we only give here some comments :

- If the strength of the repulsive term is taken as independent of the number of $\mathrm{d}$ electrons, one finds that the compressibility and the elastic moduli are proportional to the cohesive energy. This is roughly true as can be seen in figure 8 .

- Though quite simple the model do not reduce to a model of central forces between atoms ; as a conse-

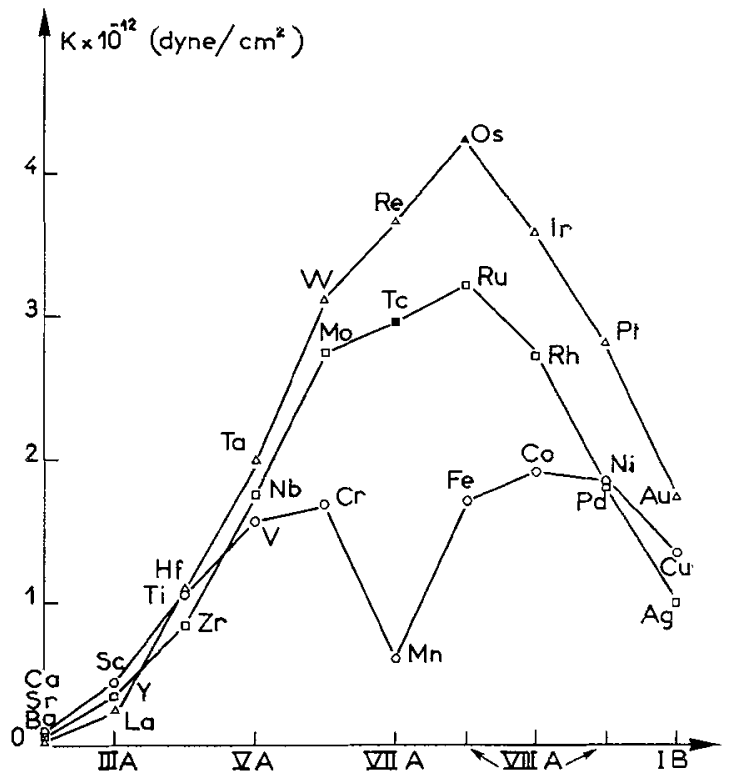

Fig. 8. - Experimental values of the bulk modulus $K$. 
quence deviations from the Cauchy relations are found, in good agreement with the experimental data.

- Finally the whole scheme seems to be consistent, i. e. a small number of parameters is sufficient to explain the variations of several quantities : cohesive energy, bulk modulus, shear moduli, deviations from the Cauchy relations, etc...

3.5. Conclusion. - A lot of other problems can be studied in a similar way. Let us just mention the calculation of surfaces energies [3] and of ponctual defects [20]. It turns out in these cases that the brokenbond model is justified as a first approximation. Breaking a bond is roughly equivalent to an energy loss of $2 E_{\mathrm{c}} / \mathrm{Nz}$ where $N$ is the number of atoms. Unfortunately just as it is, the model cannot be applied to situations involving large displacements of atoms such as those found in dislocations, grain boundaries, etc...; large displacements of atoms implying charge transfers which must be taken into account in a self-consistent way.

4. Covalent crystals. - 4.1 Introduction. - The tight-binding approximation has been widely applied to the study of covalent crystals or molecules. At first the validity of the method is perhaps more doubtful than in the case of transition metals. We are concerned here with $s$ and $p$ orbitals which are less concentrated on the atoms than the $d$ ones. Nevertheless, with some refinements the method can reasonably account for the electronic spectra of typical covalent crystals such as diamond, silicium or germanium [21]. This is mainly true for the valence band of these semiconductors which contains bonding states, the conduction band being more appropriately described by orthogonalized plane waves. When dealing with cohesive energies, we are mainly interested by the occupied states of the valence band and there is some hope that the tight-binding approximation should give at least good qualitative results. The simplest tightbinding scheme is then to build up hybridized sp orbitals in the usual way and to take only into account the more important $\sigma$ transfer integrals. This model was first studied by Leman [22] ; some years ago Thorpe and Weaire have shown that it can be successfully used to describe amorphous semiconductors which respect the tetravalent local environment [23].

\subsection{The Leman-Thorpe-Weaire MODEl. -} 4.2.1 Description of the model. - For definiteness, let us consider a tetravalent crystal like diamond. We first define the usual hybridized sp orbitals on site $i$

$$
\begin{aligned}
& \sqrt{4}|i, 1>=| \mathrm{s}\rangle+\left|p_{x}\right\rangle+\left|p_{y}\right\rangle+\left|p_{z}\right\rangle \\
& \sqrt{4}|i, 2\rangle=|\mathrm{s}\rangle+\left|p_{x}\right\rangle-\left|p_{y}\right\rangle-\left|p_{z}\right\rangle \\
& \sqrt{4}|i, 3>=| \mathrm{s}>-\left|p_{x}\right\rangle+\left|p_{y}\right\rangle-\left|p_{z}\right\rangle \\
& \sqrt{4}|i, 4>=| \mathrm{s}\rangle-\left|p_{x}\right\rangle-\left|p_{y}\right\rangle+\left|p_{z}\right\rangle .
\end{aligned}
$$

The orbital $|i, 1\rangle$ points along a bond toward a first neighbour $i^{\prime}$. On $i^{\prime}$ we define similar orbitals by performing an inversion $(x \rightarrow-x, \ldots)$ in such a way that the new orbital $\left|i^{\prime}, 1\right\rangle$ points toward $|i, 1\rangle$. To a given bond $J$ are associated two orbitals $|i, J\rangle$ and $\left|i^{\prime}, J\right\rangle$. The simplest tight-binding approximation is to neglect all transfer integrals but the following one :

$$
\beta=\left\langle i, J\left|V_{i}\right| i^{\prime}, J\right\rangle .
$$

But here we start from two different atomic levels $E_{\mathrm{s}}$ and $E_{\mathrm{p}}$. With the new basis (4.1), the intra-atomic integrals are given by:

$$
\begin{aligned}
& <i, J|H| i, J\rangle=\frac{1}{4}\left(E_{\mathrm{s}}+3 E_{\mathrm{p}}\right) \\
& \left.<i, J|H| i, J^{\prime}\right\rangle=\frac{1}{4}\left(E_{\mathrm{s}}-E_{\mathrm{p}}\right) \quad J^{\prime} \neq J .
\end{aligned}
$$

We fix the zero of energies: $E_{\mathrm{s}}+3 E_{\mathrm{p}}=0$, and finally we are left with two different negative integrals :

$$
\begin{aligned}
& \Delta=\frac{1}{4}\left(E_{\mathrm{s}}-E_{\mathrm{p}}\right)=\left\langle i, J|H| i, J^{\prime}\right\rangle \quad J^{\prime} \neq J \\
& \beta=\left\langle i, J|H| i^{\prime}, J\right\rangle \quad i^{\prime} \neq i .
\end{aligned}
$$

4.2.2 Density of states [23], [24]. - When $\beta=0$, we have the atomic limit with two levels, $E=E_{\mathrm{s}}$ and $E=E_{\mathrm{p}}$. The opposite situation $\Delta=0$ is more interesting. In this case we have :

$$
H^{2}|i, J\rangle=\beta^{2}|i, J\rangle \text { for all }|i, J\rangle
$$

and we have again two levels $E= \pm \beta$ corresponding to pure bonding and antibonding states. This remarkable property can be seen to be related to the odd character of the sp orbitals. Starting from each limit, we expect a progressive broadening of the discrete levels. In fact, in this model, there is always a gap. Numerous and various proofs of this result are available in the litterature (see for example [23] and [24]). Another surprising consequence of the model is the persistence of discrete levels in the density of states associated with pure $p$ states. Let us write the state $|\psi\rangle$ as :

$$
\left|\psi>=\sum_{i, J} a_{i J}\right| i, J>
$$

and let us assume that :

$$
\begin{aligned}
\sum_{J} a_{i J} & =0 & & \text { for all } i \\
a_{i J} & = \pm a_{i^{\prime} J} & & \text { for all } J .
\end{aligned}
$$

By inspection it is then realized that these states are the announced $p$ eigenstates of $H$ :

$$
H|\psi>=(-\Delta \pm \beta)| \psi>\text {. }
$$

Eq. (4.7) implies $3 N$ conditions, $N$ being the number of atoms. The total number of states is $4 N$ (spin not 
included) and therefore to each discrete $p$ level is associated one state per atom. Finally a further analysis show that the spectrum associated with the two remaining states is completely determined by the solution of a simpler problem. Let $\mathcal{H}$ be the hamiltonian of a hypothetical system with the same lattice, but with a single s state $\mid i>$ at each site $i$ :

$$
\mathscr{H}=\sum_{i^{\prime} \neq i}^{\prime}|i\rangle\left\langle i^{\prime}\right|
$$

and let $\varepsilon$ be the eigenvalues of $\mathscr{H}$, then the continuous spectrum of $H$ is given by :

$$
E=\Delta \pm \sqrt{4 \Delta^{2}+\beta^{2}+\beta \Delta \varepsilon} .
$$

This is the Thorpe-Weaire theorem which gives a complete treatment of the degeneracy of the sp states. Two cases can be distinguished:

$$
\begin{aligned}
& \text { - atomic limit } \left.\Delta / \beta>\frac{1}{2} \text { (Fig. } 9 a\right) \\
& \text { - covalent limit } \left.\Delta / \beta<\frac{1}{2} \text { (Fig. } 9 c\right) .
\end{aligned}
$$

The intermediate case is described by figure $9 b$. Each continuous subband contains one state per atom.

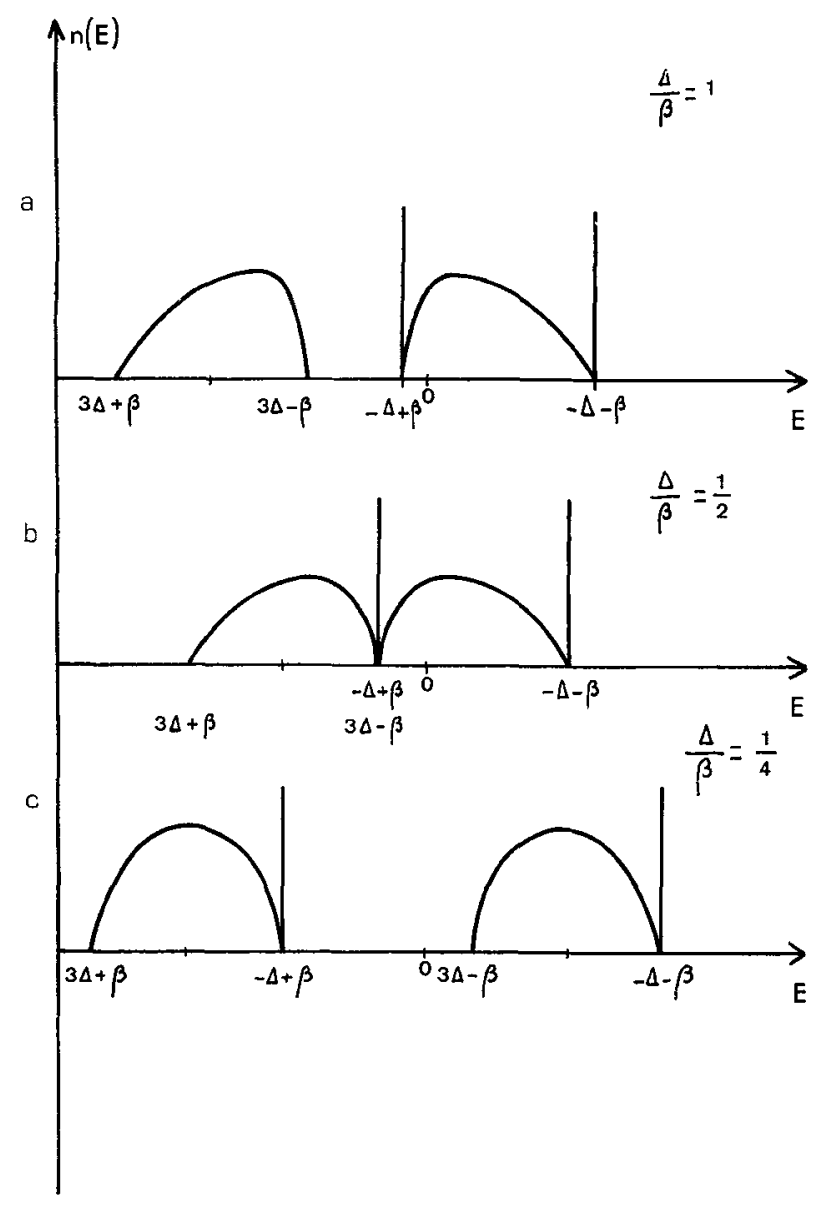

FIG. 9. - Sketch of the density of states in the Leman-ThorpeWeaire model $; \Delta / \beta=1$ : atomic regime; $\Delta / \beta=1 / 4$ : covalent regime.
Therefore as expected the whole valence band contains one state in the atomic limit and two states in the covalent limit. In all cases the width of the gap is given by :

$$
g=2|\beta-2 \Delta|
$$

The model is of course oversimplified ; in a more realistic treatment the $\mathrm{p}$ levels are broadened, and there is a regime where the gap disappears. Yet it gives a clear qualitative description of the nature of the electronic states.

4.3 Moments [25], [26]. - Let us now use our general formalism and write down the moments :

$$
\begin{array}{r}
\mu_{n}=\frac{1}{4 N} \sum_{\substack{i_{1}, \ldots, i_{n} \\
J_{1}, \ldots, J_{n}}}<i_{1}, J_{1}|H| i_{2}, J_{2}>\cdots \\
\cdots<i_{n}, J_{n}|H| i_{1}, J_{1}>
\end{array}
$$

Two kinds of steps are allowed : intra-atomic steps associated with $\Delta$, and interatomic steps associated with $\beta$. Hence :

$$
\mu_{n}=\frac{1}{4} P_{n}^{t} \beta^{n-t} \Delta^{t}
$$

where $P_{n}^{t}$ is the the number of paths including $t$ intraatomic steps. The first moments are then given by :

$$
\mu_{1}=0 ; \quad \mu_{2}=3 \Delta^{2}+\beta^{2} \text {. }
$$

In the simple model used here the information provided by the moments is less accurate than that obtained directly in $\S 4.2$, but as previously noticed the moments technique can easily be generalized to more realistic models. In fact when dealing with actual semiconductors, we are mostly interested by the covalent limit $\Delta / \beta \ll 1$ for which a perturbation expansion can be used. Up to second order terms one finds :

$$
\mu_{2 n} \simeq \beta^{2 n}\left[1+3 n^{2}\left(\frac{\Delta}{\beta}\right)^{2}\right] ; \quad \mu_{2 n+1} \simeq 0
$$

$\mu_{2 n}=\beta^{2 n}$ would correspond to the two discrete levels of the covalent limit; the corrections describe the broadening of these levels.

4.4 Cohestve ENERGY. - We compare the band energy to the energy of free atoms in the configuration $s^{2} p^{n-1}$. The atomic energy is then :

$$
E_{0}=2 E_{\mathrm{s}}+2 E_{\mathrm{p}}=4 \Delta \text {. }
$$

When the spin degeneracy is taken into account, there is just four available states in the valence band (in the covalent limit) which is then full whereas the conduction band is empty. A development of the band energy $E_{\mathrm{b}}$ as a function of $\Delta / \beta$ gives:

$$
E_{\mathrm{b}} \simeq 4 \beta\left(1-\frac{3}{4}\left(\frac{\Delta}{\beta}\right)^{2}\right)
$$


which yields the cohesive energy (remember that both $\Delta$ and $\beta$ are negative) :

$$
E_{\mathrm{c}}=4\left(|\beta|-|\Delta|+\frac{3}{4} \frac{\Delta^{2}}{|\beta|}\right)+0\left(\frac{\Delta^{3}}{\beta^{3}}\right) .
$$

The first two terms in $E_{\mathrm{c}}$ give the well known balance between the promotion energy $4|\Delta|$ and the bonding energy $4|\beta|$. Writing $E_{\mathrm{c}}$ as :

$$
E_{\mathrm{c}}=\frac{1}{2} \sum_{\text {bonds }} E_{\mathrm{B}}
$$

where $E_{\mathrm{B}}$ is an energy per bond :

$$
E_{\mathrm{B}} \simeq 2|\beta-\Delta|+\frac{3}{2} \frac{\Delta^{2}}{|\beta|} \text {. }
$$

From the experimental values of $\mathrm{g}$ and $\Delta$ we can obtain an estimate of $\beta$ and therefore of the cohesive energy. In table II the results are given for $\mathrm{C}, \mathrm{Si}$ and $\mathrm{Ge}$. As far as the order of magnitude is concerned the agreement with the experimental values is not too bad. The parameter $\Delta / \beta$ is about 0.25 which justifies the use of a perturbation expansion.

\section{TABLE II}

Experimental values of the gap $g$, of $|\Delta|$ and $E_{\mathrm{c}}$ and calculated values of $|\beta|$ and $E_{\mathrm{c}}$ (energies in $\mathrm{eV}$ ) from reference [26].

$\begin{array}{cccccc} & \mathrm{g} & |\Delta| & |\beta| & E_{\mathrm{c}}(\mathrm{th}) & E_{\mathrm{c}}(\mathrm{exp}) \\ \mathrm{C} & 5.4 & 2.1 & 6.9 & - & - \\ \mathrm{Si} & 1.2 & 1.2 & 3.0 & 4.6 & 7.4 \\ \mathrm{Ge} & 0.7 & 1.5 & 3.35 & 4.7 & 4.6\end{array}$

4.5 ENERGY OF DEFECTS AND OF STACKING FAULTS. The model of broken bonds is exact when keeping only the first term in (4.20). This enables us to estimate the energy of various defects. For example if a bond is not saturated, the energy $E_{\mathrm{B}} / 2$ is lost, but the electron which escapes goes into a $\mathrm{sp}^{n}$ orbital. As a result the net energy loss is $|\beta|$. In the same way the energy of a neutral vacancy is roughly equal to $4|\beta|$, etc... (see Lannoo and Friedel [26]).

Let us now compare the energies of different crystalline structures. We know that the diamond lattice is a FCC lattice with two atoms per cell. The two FCC sublattices correspond through a translation along the stacking direction (111). Similar covalent structures can be obtained from any compact structure ; in particular from the HCP structure we obtain the würtzite structure. There is a direct correspondence indeed between the paths in each kind of structure [27]. In particular the number of closed paths of $n$ steps is also the same for any covalent structure generated in this way. In the Leman-Thorpe-Weaire model, the corresponding stacking fault energy is therefore zero. This means that we have to introduce other parameters in the model, namely the transfer integrals between orbitals pointing along different directions. In terms of moments the difference between the structures will appear in $\mu_{6}$ which is directly related to the first selfavoiding paths. If we remember the discussion concerning transition metals, we can assert that the relative difference $\delta E_{\mathrm{c}} / E_{\mathrm{c}}$ will be very small, of the order of $10^{-3}-10^{-2}$, leading to stacking fault energies about $100 \mathrm{erg} / \mathrm{cm}^{2}$, in good agreement with the experimental data and other estimates by Chen and Falicov who used a pseudopotential theory [28].

4.6 CONCLUSION. - Compounds semiconductors can also be dealt with in the previous model by introducing an ionicity parameter [25], [29], [30]. Clearly the calculations of cohesive energies become more difficult in this case. As a matter of fact the use of eq. (3.12) for calculating the cohesive energy of covalent crystals is nothing but a rough approximation. The justifications which can be given in the case of transition metals are less convincing here mainly because we are at the border line of the validity range of the tight-binding approximation. On the other hand the advantages of the method are not negligible; the method is simple and give quite reliable qualitative informations.

\section{References}

[1] Seitz, F., The Modern Theory of Solids (McGraw-Hill), 1940.

[2] Heine, V. and Wearre, D., Sol. Stat. Phys. 24 (1970) 250.

[3] Cyrot-Lackmann, F., Adv. Phys. 16 (1967) 393.

- J. Physique Colloq. 31 (1970) C 1-67.

- J. Physique Colloq. 33 (1972) C 3-167.

- J. Phys. C: Solid State Phys. 5 (1972) 300

[4] Gaspard, J. P. and Cyrot-Lackmann, F., J. Phys. C: Solid State Phys. 6 (1973) 3077

[5] Haydock, R., Heine, V. and Kelly, M. J., J. Phys. C : Solid State Phys. 5 (1972) 2845.

[6] Desjonqueres, M. C. and Cyrot-Lackmann, F., to be published.

[7] Ducastelle, F. and Cyrot-Lackmann, F., J. Phys. Chem. Solids 32 (1971) 285.
[8] Friedel, J., The Physics of Metals, J. M. Ziman ed. (Cambridge University Press) 1969, 340.

[9] Ducastelle, F. and Cyrot-Lackmann, F., J. Phys. Chem. Solids 31 (1970) 1295.

Ducastelle, F., Thesis Orsay, 1972, ONERA publication $\mathrm{n}^{0} 144$.

[10] Slater, J. C. and Koster, G., Phys. Rev. 94 (1954) 1498.

[11] Dimmock, J. O., Sol. Stat. Phys. 26 (1971) 103.

[12] Gautier, F., Private communication.

[13] Kaufman, L., Phase Stability in Metals and Alloys, Battelle Institute Materials Science Colloquia (McGraw-Hill) 1967.

[14] Deegan, R. A., J. Phys. C : Solid State Phys. 2 (1968) 763. 
Dalton, N. W. and Deegan, R. A., J. Phys. C : Solid State Phys. 2 (1969) 2369.

Pettrfor, D. G., J. Phys. C : Solid State Phys. 3 (1970) 366. [15] JaMEs, H. M., Phys. Rev. 164 (1967) 1153.

[16] DuCastelle, F., Unpublished.

[17] Smallman, R. E., Dillamore, I. L. and Dobson, P. S., J. Physique Colloq. 27 (1966) C 3-94.

Latanison, R. M. and Ruff, A. W., J. Appl. Phys. 40 (1970) 2716.

[18] Tisone, T. C., Acta Met. 21 (1973) 229.

[19] Ducastelle, F., J. Physique 31 (1970) 1055.

[20] GerL, M., J. Phys. Chem. Solids 31 (1971) 315.

Lannoo, M. and Lenglart, P., J. Phys. Chem. Solids 32 (1971) 637.

Allan, G. and Lenglart, P., J. Physique Colloq. 31 (1970) C 1-93.

Allan, G. and Lannoo, M., Surface Sci. 40 (1973) 375.
[21] HaLl, G. G., Phil. Mag. 3 (1958) 429. Stocker, D., Proc. R. Soc. A 270 (1962) 397.

[22] Leman, G., Annls. de Phys. 18 (1963) 1.

[23] Wealre, D. and Thorpe, M. F., Phys. Rev. B 4 (1971) 2508. Thorpe, M. F. and Weaire, D., Phys. Rev. B 4 (1971) 3518. Thorpe, M. F., Weaire, D. and Alben, R., Phys. Rev. 34 (1973) 115.

[24] Friedel, J. and Lannoo, M., J. Physique 34 (1973) 115 and erratum to be published.

[25] Friedel, J., J. Less Common Metals 13 (1972) 294.

[26] Friedel, J. and Lannoo, M., J. Physique 34 (1973) 483.

[27] Thorpe, M. F., J. Math. Phys. 13 (1972) 294.

[28] ChEN, L. J. and Falicov, L. M., Phil. Mag. 29 (1974) 1; Phil. Mag. 29 (1974) 1133.

[29] Straley, J. P., Phys. Rev. B 6 (1972) 4086.

[30] Harrison, W. A., Phys. Rev. B 8 (1973) 4487. 